\title{
Functional-Voxel Method in Problems of Geometric Modeling of Thermal Characteristics of Objects*
}

\author{
Aleksandr Plaksin ${ }^{1[0000-0001-9390-8322]}$, Alexey Tolok ${ }^{1[0000-0002-7257-9029]}$, \\ ${ }^{1}$ Laboratory of Computer Graphics, V.A. Trapeznikov Institute of Control Science of Russian \\ Academy of Sciences, 65 Profsoyuznaya street, Moscow, 117997, Russia \\ a.m.plaksin@gmail.com, tolok61@mail.ru
}

\begin{abstract}
The paper presents an approach developed on the basis of the functional voxel method to the geometric representation of the thermal expansion of objects and temperature stresses in a material when exposed to a surface of a heat source. A discrete geometric law of a single temperature stress in an isotropic heat-conducting body is derived, applicable in the concept of functional voxel modeling. Based on this law, functional-voxel models of thermal stress are developed for a single and distributed application of a heat source. Algorithms of functional-voxel modeling of temperature stress and expansion in the case of distributed thermal loading are presented, which make it possible to construct a loading region of a complex configuration, uniformly form a contour (surface) after material expansion and obtain information about changes in the length (volume) of products. The advantages of the proposed functional-voxel approach to modeling thermal expansion and stress over approaches based on the FEM are substantiated.
\end{abstract}

Keywords: Discrete Geometric Model, Finite Element Method (FEM), Functional Voxel Method (FVM), Temperature Stress, Thermal Expansion.

\section{Introduction}

Modern automated solutions to the problem of calculating thermal characteristics are based on solving differential equations using numerical methods using the finite element method, which is a complex computational problem [7]. To apply this approach in the case of dynamic calculations of contact loads, it is necessary to constantly reallocate the position of the nodes of the finite element grid, which requires powerful computing resources. Moreover, it is a difficult task to include additional criteria in the calculation scheme that affect the change in the geometric shape of the modeled object. The solution of the presented problems is reduced to simplifying the problem statement,

Copyright c 2020 for this paper by its authors. Use permitted under Creative Commons License Attribution 4.0 International (CC BY 4.0).

Supported by V.A. Trapeznikov Institute of Control Science of Russian 
which entails a loss of accuracy of calculations as a result of neglecting various conditions.

Solving the problem of finding the circumference contour of the processing object, taking into account the influence of differential properties of physical processes, is an urgent task of automated calculations. The method of functional-voxel modeling is one of the possible approaches to the complex solution of this problem [2-10], due to the possibility of linking the analytical formulations of physical laws applicable at a given point in a single context of the entire discrete space under consideration. The use of the functional voxel method simplifies the calculations by reducing the calculated expression to a linear polynomial that describes the local functional dependence at a specific point in the simulated space.

Functional-voxel method has high calculated applicability in solving analytical modeling problems. This is achieved by the possibility of quick access to the differential and integral characteristics of the modeled function at each point by means of local geometric characteristics stored in multidimensional graphic images [2 - 10].

Thus, the application of the functional-voxel method to the solution of the problem posed will make it possible to have information on the stressed and expanded state of the body at each of its points in computer modeling of the geometric characteristics of thermal stress and thermal expansion.

\section{Functional-Voxel Model of Temperature Stress}

Consider the point application of the thermal load $\theta$ to an isotropic heat-conducting body. The heat applied to the body spreads evenly in the depths of the material in all directions [11], which is geometrically expressed in the representation of the heat distribution region as a sphere in the three-dimensional case and as a circle in the twodimensional $[1,12]$. Thus, the value of the function of the applied heat load changes as the applied heat is distributed over the surface area of the formed sphere:

$$
\sigma_{\Theta f}=\frac{\Theta}{4 \pi R^{2}},
$$

The presented expression will describe the distribution of heat when heat is applied inside the material, but from a practical point of view, it is necessary to consider the application of heat to the surface of a solid. In the case of an isotropic heat-conducting body under the surface influence of heat, the formed heat distribution sphere will be cut off, thus forming a hemisphere, the surface area of which is two times smaller than the surface area of the sphere:

$$
\sigma_{\Theta f}=\frac{\Theta}{2 \pi R^{2}}
$$

From the general equation of a sphere (2), it is possible to express its radius $\mathrm{R}$ :

$$
R=\sqrt[2]{x^{2}+y^{2}+z^{2}} .
$$


However, it is necessary to take into account the situation in which the radius of the sphere in the denominator of the expression is zero. There is also a situation when the calculated value $\sigma_{\theta f}$ exceeds the value of the applied heat load $\theta$ at $1 \frac{1}{\sqrt{2 \pi}}>R \geq 0$ in the case of considering a continuous representation of this propagation law. It is necessary to make a transition to its discrete representation.

The heat source is applied pointwise, then a sphere with a surface area of one is the abstract "unit" application area:

$$
\sigma_{\theta f}=\frac{\Theta}{S^{1}}=\Theta, \text { где } S^{1}=1 .
$$

From $S^{1}=4 \pi R^{2}$ we obtain $R=\frac{1}{2 \sqrt{\pi}}$. To describe a discrete law on an entire geometric body, it is necessary to take a discrete parameter that describes the distance from a certain point of application of heat to the studied point of the body. We denote it as $\Delta R_{i}$ $\mathrm{i}$-th application point $\left(x_{i}, y_{i}, z_{i}\right)$. Then, the calculated area is represented as $S_{i}=4 \pi\left(R+\Delta R_{i}\right)^{2}=4 \pi\left(\frac{1}{2 \sqrt{\pi}}+\Delta R_{i}\right)^{2}=1+4 \sqrt{\pi} \Delta R_{i}+4 \pi \Delta R_{i}{ }^{2}$. Thus, the discrete geometric law of temperature stress at some $\mathrm{i}$-th point will have the form:

$$
\sigma_{i}=f\left(x_{i}, y_{i}, z_{i}\right)=\frac{\Theta}{1+4 \sqrt{\pi} \Delta R_{i}+4 \pi \Delta R_{i}^{2}},
$$

where $\Delta R_{i}=\sqrt{x_{i}^{2}+y_{i}^{2}+z_{i}^{2}}$ provided that the origin of the coordinate system is located at the point of application of heat.

The presented expression geometrically partially corresponds to a quadratic hyperbole. The calculated value of $\sigma_{i}$ will coincide with the applied heat load $\theta$ if the discrete parameter $\Delta R_{i}$ is equal to zero. With a single applied heat load, the described law takes the form illustrated in Figure 1, which also shows the subfunctional space that is related in volume to the heat load applied to the body.

In the future, this temperature volume can be used in modeling problems in the calculations, which circumvents the need to use the physical concept of temperature.
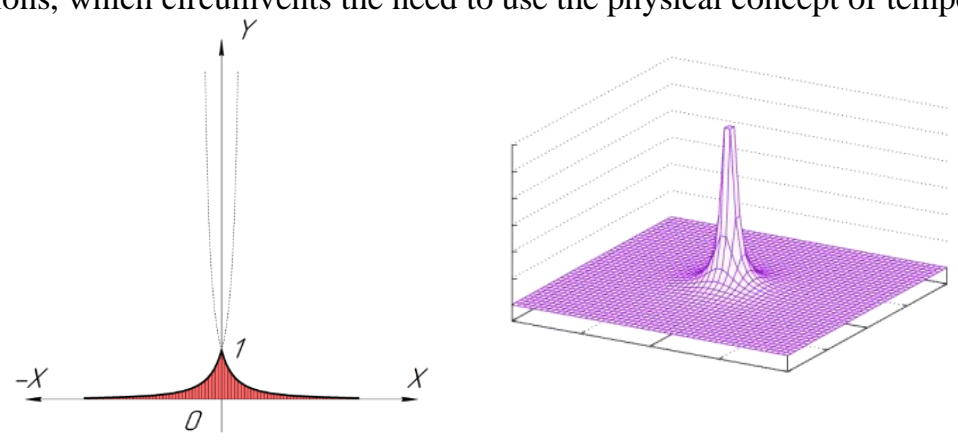

Fig. 1. The geometric law of temperature stress.

The ratio between the applied heat load and the distributed heat will be: 
4 A.Plaksin, A. Tolok

$$
\sigma_{\Theta f}=k \sigma_{V f}
$$

To determine the indicated equivalent volume of the temperature stress field, it is necessary to calculate the quadruple integral of the form:

$$
\sigma_{V i}=\int_{0}^{\Theta} \int_{0}^{l} \int_{0}^{w} \int_{0}^{h} \frac{\Theta}{1+4 \sqrt{\pi} \Delta R_{i}+4 \pi \Delta R_{i}{ }^{2}} d x d y d z d u,
$$

where $l, w$ and $h$ are the size of the studied area

The presented expression is applied in the case of research in three-dimensional space. To consider the two-dimensional case, it suffices to consider a cube, in the increased space of which the value of the function corresponds, i.e., temperature.

The necessary calculations are carried out by means of the functional-voxel method, according to the technique proposed in [13]:

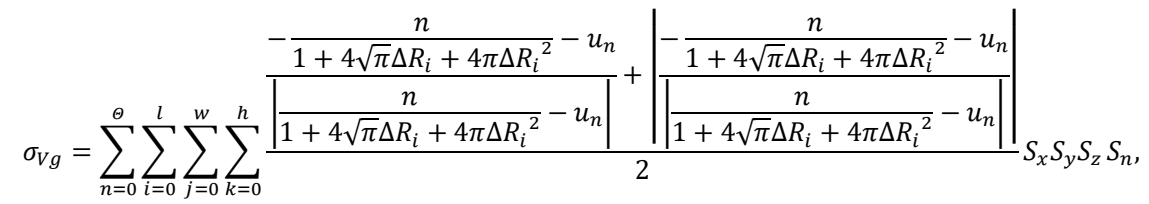

where $S_{x}, S_{y}, S_{z}$ и $S_{n}$ are the discretization of the voxel space, and $\Delta R=\sqrt{\left(i S_{x}\right)^{2}+\left(j S_{y}\right)^{2}+\left(k S_{z}\right)^{2}}$.

The solution of both equations leads to similar results, allowing us to conclude their equivalence. In the case of a single thermal effect in a space of $100 \times 100 \times 100$, the coefficient is:

$$
k=\frac{1}{30} .
$$

As a result, we obtain the equilibrium equation of volume distribution with which it is possible to supplement the developed model of unit temperature stress. When simulating heat propagation, this equation will allow one to determine the heating of the application point $\mathrm{k}$ times less than the applied thermal loading.

The obtained discrete geometric law can be applied to carry out functional-voxel modeling of temperature stress in a solid isotropic heat-conducting body with a single and distributed application of a heat source [6,7].

Figures 2 and 3 show a comparison of the visualization of the functional-voxel models (FVM) with a similar study conducted by the finite element method (FEM). As can be seen from the figures, the proposed approach allows us to obtain a geometrically adequate law of heat distribution. 


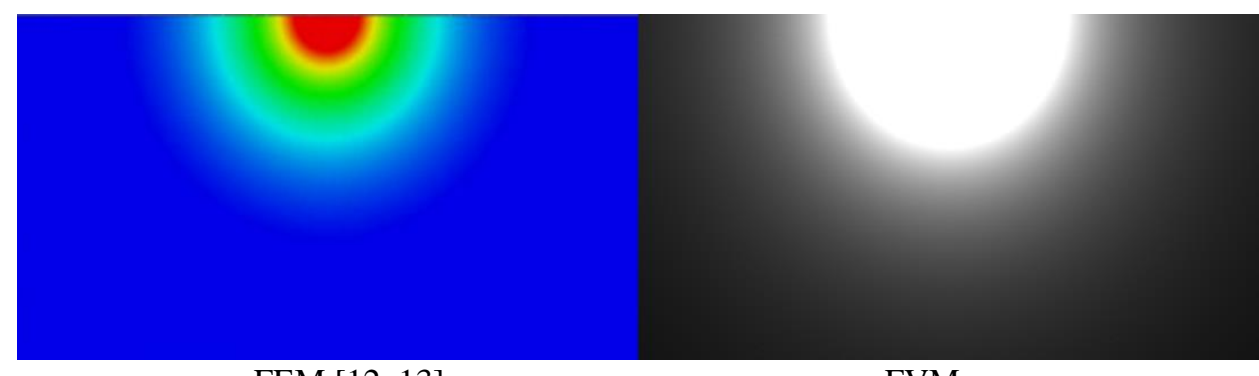

FEM $[12,13]$

FVM

Fig. 2. Comparison of the temperature stress distribution of the FEM and the FVM when applying a load at a point

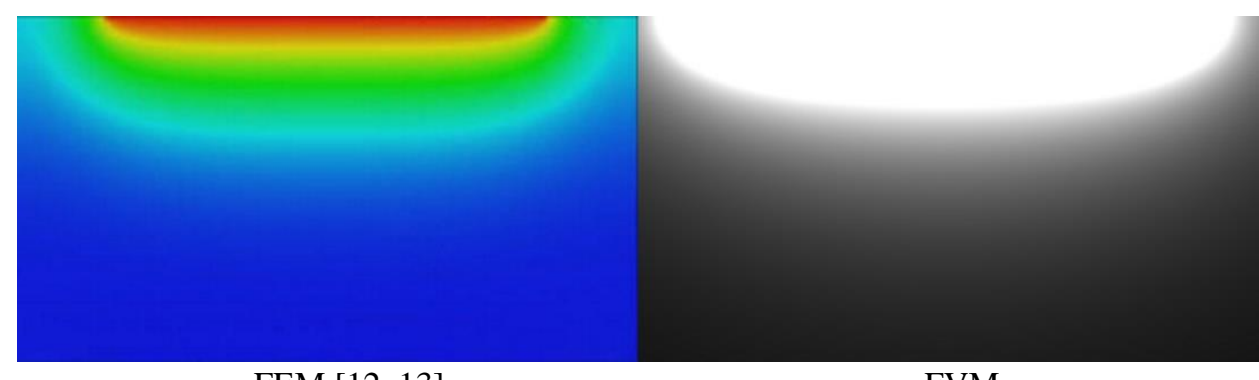

FEM $[12,13]$

FVM

Fig. 3. Comparison of the temperature stress distribution of the FEM and the FVM when applying a distributed load.

\section{Functional-Voxel Model of Thermal Expansion}

The interpreted law of the classical theory of thermal expansion for a discrete model will be: $\Delta V_{i}=\alpha T_{i} V_{i}$, where $\alpha$ is the coefficient of thermal expansion, $T_{i}$ is the temperature stress at the $\mathrm{i}$-th point, and $V_{i}$ is the volume of the sphere formed by an increment of the radius $\Delta R_{i}$.

Then, to obtain a discrete geometric model of thermal expansion of the surface, it is necessary to calculate the increment of coordinates at the i-th point (for example $\Delta z_{i}$ ). To do this, it is necessary to divide the obtained volume $\Delta \mathrm{V}_{i}$ into a discrete model for determining the current area of the circle $K_{i}$ as a platform that is incremented by the radius $\Delta R_{i}$ :

$$
\begin{gathered}
S^{1}=\pi R^{2}=1, \text { and therefore } R=1 / \sqrt{\pi} ; \\
S_{i}=\pi\left(R+\Delta R_{i}\right)^{2}=\pi\left(\left(\frac{1}{\sqrt{\pi}}\right)^{2}+2 \frac{1}{\sqrt{\pi}} \Delta R+\Delta R^{2}\right)=1+2 \sqrt{\pi} \Delta R_{i}+\pi \Delta R_{i}^{2} ; \\
\Delta z_{i}=\frac{\Delta \mathrm{V}_{i}}{1+2 \sqrt{\pi} \Delta R_{i}+\pi \Delta R_{i}^{2}},
\end{gathered}
$$


where $\Delta V_{i}=\alpha T_{i} V_{i}$.

The volume $\Delta \mathrm{V}_{i}$ is determined similarly to the considered $S_{i}$ with the expression of the accumulated volume $V_{i}$ :

$$
\begin{gathered}
V^{1}=\frac{4}{3} \pi R^{3}=1 \text { and therefore } R=\sqrt[3]{\frac{3}{4 \pi}} ; \\
V_{i}=\frac{4 \pi\left(R+\Delta R_{i}\right)^{3}}{3}=\frac{4 \pi}{3}\left(R^{3}+3 R^{2} \Delta R_{i}+3 R \Delta R_{i}^{2}+\Delta R_{i}^{3}\right)= \\
1+3 \sqrt[3]{\frac{4}{3}} \pi \Delta R_{i}+3 \sqrt[3]{\left(\frac{4}{3} \pi\right)^{2}} \Delta R_{i}^{2}+\frac{4}{3} \pi \Delta R_{i}^{3},
\end{gathered}
$$

where $\Delta R_{i}=\sqrt{x_{i}^{2}+y_{i}^{2}+z_{i}^{2}}$.

With an increase in the radius of the generatrix of the sphere, the temperature value decreases according to the hyperbolic law, which means that the obtained temperature volume at the greatest distance minimally affects the geometry of the object. From this we can conclude that the thermal expansion of the body by the volume $\Delta V_{i}$ at a specific iteration will be carried out over the entire area of the circle with a radius $\Delta R_{i}$, the value of which is the maximum remote distance, which is shown in Figure 4.

A
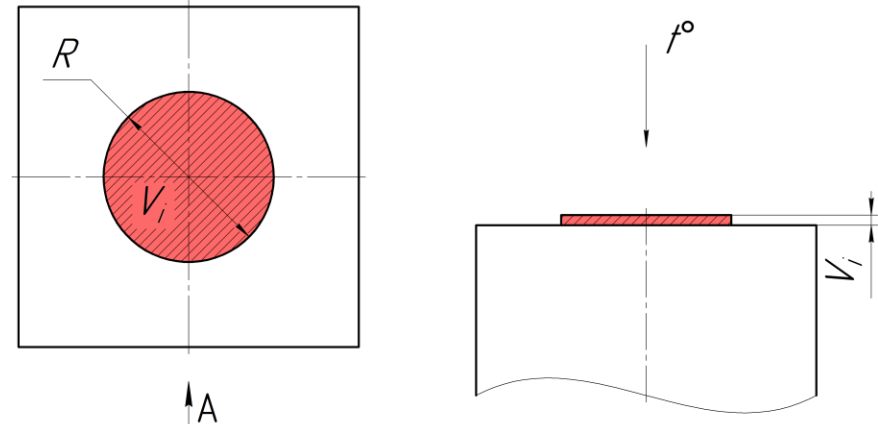

Fig. 4. The change in body volume by $\Delta V_{i}$ for the current $R$.

The presented model makes it possible to exert a point thermal effect on the surface, using the concept of an abstract "unit" site, in contrast to the finite element method, where it is necessary to use the region that makes up the element of the computational grid as the loading point. Comparison of thermal expansion results by the presented method and finite element method is shown in Figure 5, where geometric similarity of simulation results can be observed $[1,12]$. 


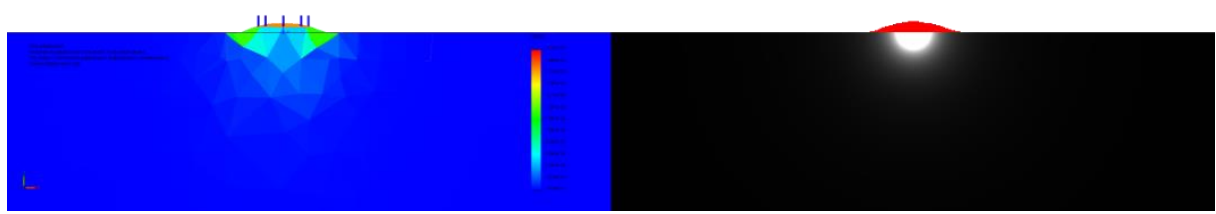

FEM [12, 13]

FVM

Fig. 5. Comparison of simulation results with the point impact of a heat source to the body surface.

The obtained model of heat distribution from a point source can be applied to obtain a model of heat distribution from a distributed application of the source to the surface of the body. In this case, the law of thermal expansion for a unit load must be applied to each point of the contact area between the surface and the heat source. Thus, we obtain a change in the volume of the current iteration $\Delta V_{i}$ for a point with the distribution over the area of a sphere with radius $\mathrm{R}$ distributed along a certain straight line $\mathrm{L}$ (see Figure 6).
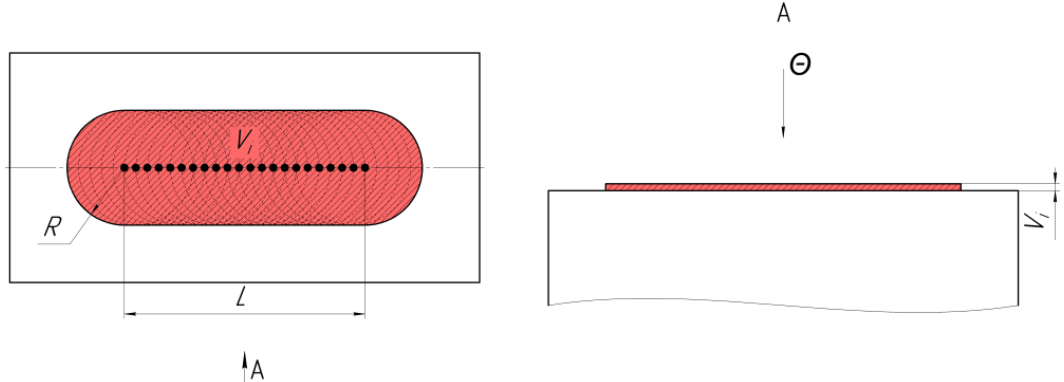

Fig. 6. Change in body volume by $\Delta V_{i}$ for the current $\mathrm{R}$ during load distribution along $\mathrm{L}$.

Figure 7 presents the simulation results of the proposed method and the finite element method, which indicate the similarity of the results of both modeling methods $[1,7]$.

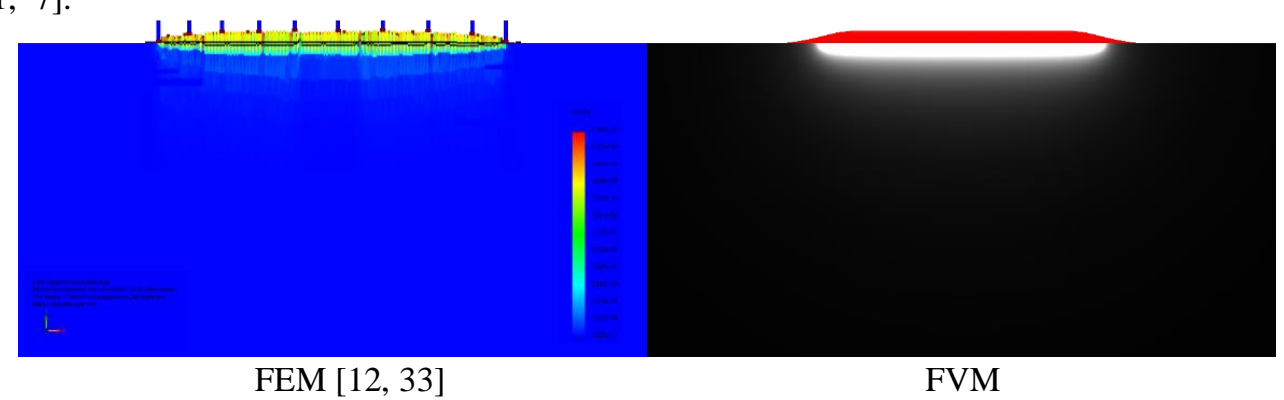

Fig. 7. Comparison of simulation results with the distributed effect of the heat source to the surface of the body.

Due to the modeling of the application of both point and distributed application of heat to the surface of a complex geometric shape [8-10], it is possible to create a full- 


\section{A.Plaksin, A. Tolok}

fledged device for accounting for thermal characteristics during the passage of a cutting tool. At the same time, the construction of complex geometric contours is realized through set-theoretic operations, and specifically, with the R-functional modeling apparatus $[2,4,8]$. Figures 8 and 9 show examples of the implementation of modeling the application of thermal influence to the contour of a part of a complex configuration with the occurring thermal expansion.

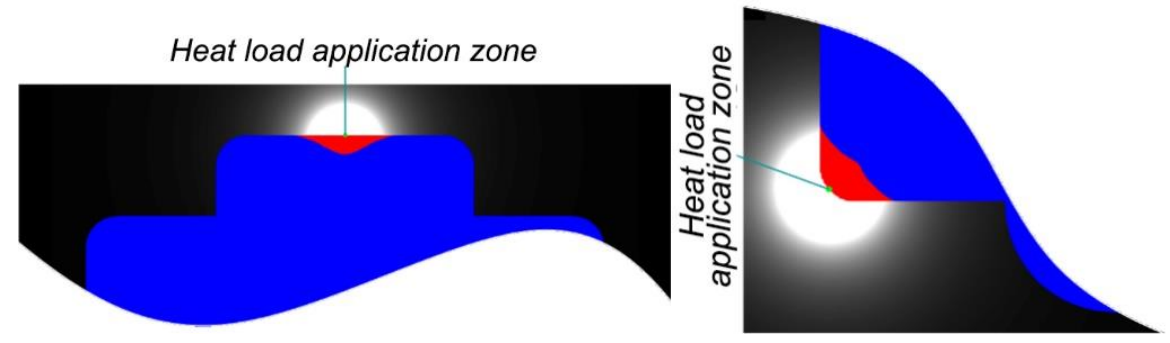

Fig. 8. Example of applying a heat load at a point in a contour section.

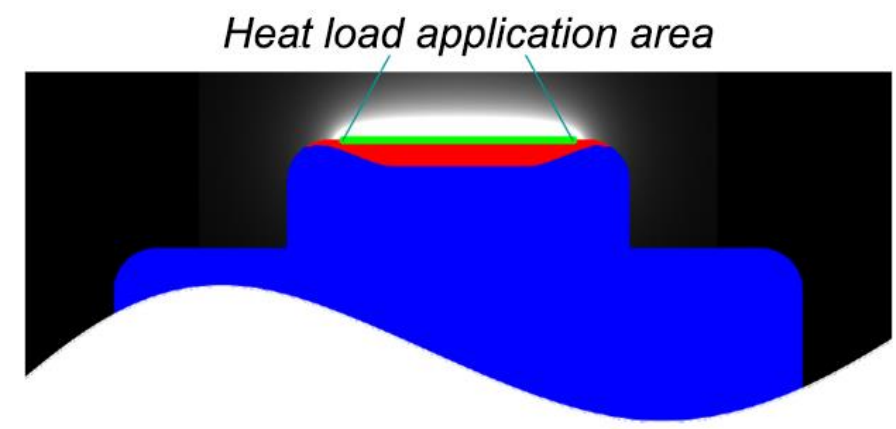

Fig. 9. Example of applying a distributed heat load to a specified section of the contour.

\section{$4 \quad$ Refine Workpiece Bypass Contour}

The developed approach can be applied to clarify the contour of the bypass of the processing tool during the machining of parts. Consider this by the example of milling on a universal milling machine of a beam workpiece. The measurement results of the actual contour obtained during processing are presented in Figure 10. In this case, the measurements were carried out after the workpiece reached thermal equilibrium. 
Functional-Voxel Method in Problems of Geometric Modeling of Thermal Characteristics... 9

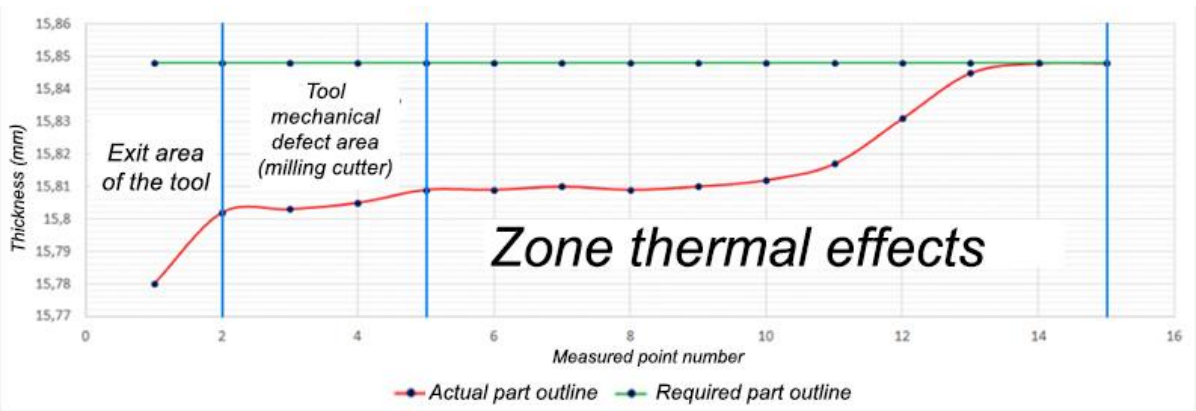

Fig. 10. Comparison of the actual and required contour of a workpiece of the beam-type after the milling operation.

To form a contour based on the functional-voxel method, it is necessary to use temperature values that affect the distortion of the shape of the workpiece. Figure 11 shows a functional image that visualizes the shape of the resulting thermal expansion of the workpiece as a result of its processing and the tool bypass circuit. Using functional voxel modeling tools, the resulting image was scaled along the abscissa for clarity.

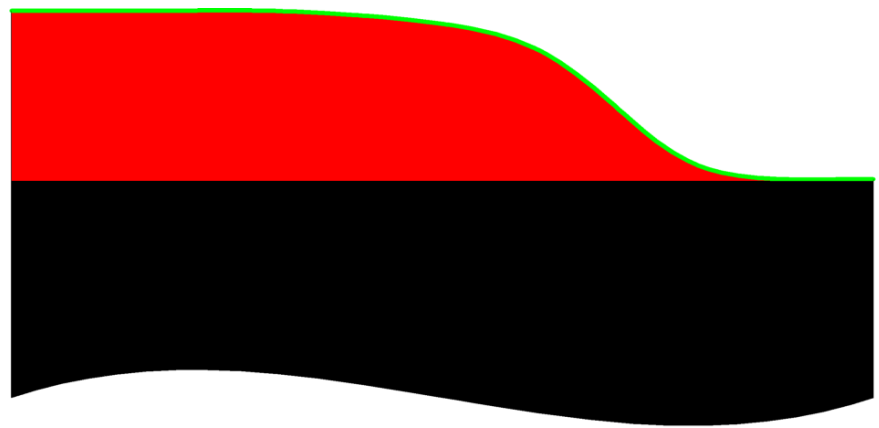

Fig. 11. The formation of the contour for the passage of the processing tool.

The circuit obtained as a result of functional voxel modeling was used to compile the control program code for a CNC milling machine for processing a workpiece of the "beam" type. Figure 12 shows the results of measurements taken at the end of processing and after the workpiece reaches thermal equilibrium, in comparison with the results of processing on a universal machine. According to the above data, we observe a more rectilinear contour of the part due to a decrease in thermal effects. 


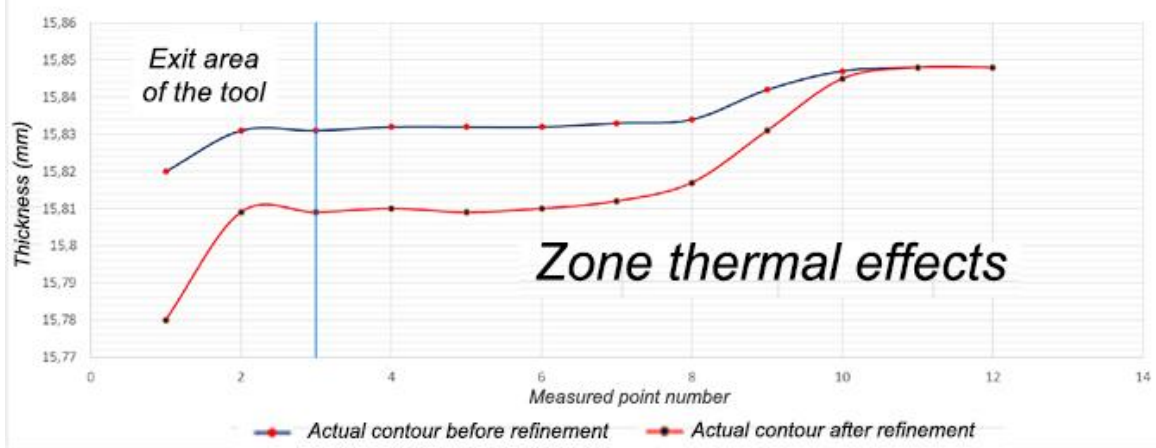

Fig. 12. Comparison of results before and after refinement of the circuit using the FVM method.

\section{References}

1. Plaksin, A. M., Sycheva A. A. Functional voxel modeling of thermal characteristics. Abstracts of the 18th international youth conference «Systems of design, technological preparation of production and management of stages of the life cycle of an industrial product», 36-36 (2018).

2. Reznikov A. N. Thermal processes in technological systems. Textbook for universities. Moscow (1990).

3. Tolok A. V. Graphic images-models in information technologies. Applied informatics 4, 3140 (2009).

4. Tolok A. V. Application of voxel models in the process of mathematical modeling automation. Automation and remote control 6, 167-180 (2009).

5. Tolok A. V. Synthesis of computer images of geometric characteristics for estimating the surface relief of a function of two variables. Collection of reports of the national Academy of Sciences of Ukraine. Mathematics, natural science, technical Sciences 4, 63-69 (2004).

6. Tolok A. V. Functional voxel method in computer modeling. Moscow (2016).

7. Gustafson R. J., Thompson D. R., Sokhansanj S. Temperature and stress analysis of corn kernel-finite element analysis. Transactions of the ASAE 4 (22), 955-960 (1979).

8. Rvachev V. L., Tolok A. V., Uvarov R. A., Sheiko T. I. New Apparoaches to Generating Equations of the Three-dimensional Loci Using R-functions. Vestn. Zaporozh. Gos. Univ (2), 119-131 (2000).

9. Tolok A. V., Myl'tsev A. M., Korogod V. L. An Algorithm of Spatial Motion along a Gradient on the Basis of M-images. Applied Geometry and Engineering Graphics 77, 85-90 (2007).

10. Tolok A. V., Tolok N. B., Loktev M. A. Modeling Function Domain for Curves Constructed Based on a Linear Combination of Basis Bernstein Polynomials. Programming and Computer Software 6 (44), 526-532 (2018).

11. Plaksin, A. M., Sycheva A. A. Modeling of thermal characteristics based on a functional voxel model with a preliminary definition of the bypass contour. Proceedings of the 13th all-Russian meeting on management issues, 3179-3185 (2019).

12. Alyamovskij A. A. SolidWorks Simulation. Engineering analysis for professionals: tasks, methods, recommendations]. Moscow, (2015). 
Functional-Voxel Method in Problems of Geometric Modeling of Thermal Characteristics... 11

13. ZHukov N. P., Majnikova N. F., Nikulin S. S., Antonov O. A. Solving problems of thermal conductivity by the finite element method. Tambov, (2014).

14. Rvachev V. L. Theory of R-functions and some of its applications. Kiev (1982).

15. Zhambalova S. B. R-functions in mathematical modeling of geometric objects. Materials of the 1st round of the International student scientific and practical conference «Automation and information technologies», (2019).

16. Kravchenko V. F., Kravchenko O. V., Churikov D. V. Application of R-functions, atomic and Wa-systems of functions in information technologies. Review. Current problems of modern education 2, 13-23 (2018).

17. Loktev M. A. Features of application of functional voxel modeling in problems of finding a path with obstacles. Information technologies in design and production 1, 45-49 (2016). 